\title{
REFLECTION POSITIVITY AND MONOTONICITY
}

\author{
ARTHUR JAFFE AND GORDON RITTER
}

\begin{abstract}
We prove general reflection positivity results for both scalar fields and Dirac fields on a Riemannian manifold, and comment on applications to quantum field theory. As another application, we prove the inequality $C_{D} \leq C_{N}$ between Dirichlet and Neumann covariance operators on a manifold with a reflection.
\end{abstract}

\section{INTRODUCTION}

Reflection positivity (RP) provides the fundamental relation between functional integration and quantization. Osterwalder and Schrader formulated this notion in an attempt to understand the special case discovered by Symanzik 17 —elaborated by Nelson [14, 13], and by many authors since - between Markov random fields and quantum fields. The Osterwalder-Schrader theory not only pertains to classical probability theory, but also makes it possible to incorporate theories with spin (fermions and gauge theory), and provides the possibility to quantize differential forms. In quantum theory it leads to an analysis of the Hamiltonian and to other symmetry groups, see for instance [5, 12, 10, 4]. RP also pertains to the framework of statistical physics on a lattice, where it leads to an analysis of the transfer matrix. As a result of the importance of RP, several different ways to understand it appear in the literature.

In this paper we analyze some properties of RP, monotonicity, and static spacetimes. In particular we analyze RP arising from the Green's function for the Laplace operator under general conditions, leading to a positive inner product distinct from the standard positive inner product given by the Green's function for the Laplacian.

We also analyze the case of a general Dirac operator compatible with time reflections. This case presents new phenomena, as the Green's function for the Euclidean Dirac operator is not positive. However, using time-reflection, we establish the existence of a positive inner product and a corresponding Hilbert space, providing a general framework for quantization in this case as well.

\section{Reflections and the Laplace Operator}

Let $M$ be a complete, connected Riemannian manifold without boundary, and with isometry group $G$. Let $U$ denote the natural unitary representation of $G$ on $L^{2}(M)$, defined on a dense domain by

$$
U_{\psi} f:=f^{\psi}=f \circ \psi^{-1} \text { for } \quad \psi \in G .
$$

Let $\nabla$ denote the Levi-civita connection on $M$ associated to the metric. Let $\Delta=\Delta_{M}=\nabla^{*} \nabla$ denote the (negative-definite) covariant Laplacian, defined initially on the domain $C_{c}^{\infty}(M)$ of smooth functions of compact support. Under our

Date: May 3, 2007. 
assumptions, it follows that $\Delta$ is essentially self-adjoint on this domain ([6]; see also [2]), and so naturally we consider the unique self-adjoint extension and use the spectral theorem accordingly. The operator $U_{\psi}$ commutes with $\Delta$, which can be seen by writing $\Delta=d^{*} d$ on 0 -forms. The resolvent $C=\left(-\Delta+m^{2}\right)^{-1}$ is a bounded operator on $L^{2}(M)$. It also follow 11 that $\left[U_{\psi}, C\right]=0$, which becomes $C f^{\psi}=(C f)^{\psi}$ in the notation of eq. (11).

For $\theta \in G$, the fixed-point set is the set

$$
M^{\theta}=\{p \in M: \theta(p)=p\} .
$$

The isometry $\theta$ is said to be a reflection if $d \theta_{p}$ is a hyperplane reflection in the tangent space for some $p \in M^{\theta}$. In this case, $M^{\theta}$ is a disjoint union of totally geodesic submanifolds, at least one of which is of codimension one [1]. Any codimension-one component of $M^{\theta}$ is called a reflection hypersurface.

To formulate a general notion of reflection positivity, let $M$ be a complete connected Riemannian manifold with a reflection $\theta$. Let $\Omega \subset M$ be a submanifold with boundary $\partial \Omega$, such that $\partial \Omega$ is contained in a union of reflection hypersurfaces. Let $f \in L^{2}(M)$ be a complex-valued function with support $\mathscr{S}_{f}$, which is of class $C^{2}$ (i.e. all second derivatives are continuous), such that

$$
\theta\left(\mathscr{S}_{f}\right) \subseteq \Omega \quad \text { and } \operatorname{vol}\left(\Omega \cap \mathscr{S}_{f}\right)=0 .
$$

We do not assume that $\theta$ is disecting, i.e. that $M \backslash M^{\theta}$ is disconnected.

Example 1. Choose coordinates $(t, \vec{x})$ on $\mathbb{R}^{d}$, and define $\mathbb{R}_{+}^{d}$ and $\mathbb{R}_{-}^{d}$ to be the half-spaces with $t \geq 0$ or $t \leq 0$ respectively. Let $f$ be such that $\mathscr{S}_{f} \subseteq \mathbb{R}_{+}^{d}$, and $\Omega \subseteq \mathbb{R}_{-}^{d}$ with $\partial \Omega \subseteq\{t=0\}$

Example 2. Let $T$ be a Riemann surface with an antiholomorphic involution $\theta$ : $T \rightarrow T$, such as $\theta(z)=1 / \bar{z}$ on the Riemann sphere. Accordingly, write $T=T_{-} \cup T_{+}$, where $T_{ \pm}$are closed, $T^{\theta}=\partial T_{ \pm}$, and $\theta: T_{+} \rightarrow T_{-}$. Let $\mathscr{S}_{f} \subseteq T_{+}$and $\Omega \subseteq T_{-}$with $\partial \Omega \subset T^{\theta}$. For the Riemann sphere, $T^{\theta}$ is the unit circle $|z|=1$.

Theorem 1. Let $M$ be complete and connected with a reflection $\theta$. Let $f$ and $\Omega$ be as above. Then

$$
0 \leq\left\langle f^{\theta}, C f\right\rangle
$$

Proof. For $u, v: M \rightarrow \mathbb{C}$, let $(u, v)=\bar{u} v \mathrm{dV}$, where $\mathrm{dV}$ denotes the natural Riemannian volume measure on $M$. Define $u=C f$ and note that, by eq. (2), $C^{-1} u^{\theta}=\left(C^{-1} u\right)^{\theta}=f^{\theta}$ has support in $\Omega$. Hence

$$
\left\langle u, f^{\theta}\right\rangle=\int_{\Omega}\left(u, C^{-1} u^{\theta}\right)=\int_{\Omega}\left[\left(u, C^{-1} u^{\theta}\right)-\left(C^{-1} u, u^{\theta}\right)\right],
$$

where the second equality holds because $C^{-1} u=f$ is zero a.e. in $\Omega$. Let $n$ denote the unit normal vector to $\partial \Omega$. Now in (4), replace $C^{-1}$ with $-\Delta+m^{2}$ and integrate by parts to find

$$
\left\langle f^{\theta}, u\right\rangle=\int_{\Omega}\left[\left(\Delta u, u^{\theta}\right)-\left(u, \Delta u^{\theta}\right)\right]=\int_{\partial \Omega}\left[u^{\theta} \nabla_{n} \bar{u}-\bar{u} \nabla_{n} u^{\theta}\right] \mathrm{dS} .
$$

For $p \in \partial \Omega$, it clear that $d \theta_{p}=d \theta_{p}^{-1}=\operatorname{diag}(-1,1, \ldots, 1)$ in a coordinate basis for $T_{p} M$ where the first coordinate is in the direction of $n_{p}$ and the other directions

\footnotetext{
${ }^{1}$ See for instance [11, Theorem III.6.5].
} 
are tangential to $\partial \Omega$. Hence $\left(\nabla_{n} u^{\theta}\right)_{p}=-\left(\nabla_{n} u\right)_{p}$. Using this (and the identity $u^{\theta}=u$ on $\partial \Omega$ ) to simplify the second term in (5), we have

$$
\left\langle f^{\theta}, C f\right\rangle=2 \Re \int_{\partial \Omega} u \nabla_{n} \bar{u} \mathrm{dS}
$$

where $\Re$ denotes the real part. We now show that this quantity is real (and positive), completing the proof.

$$
\begin{aligned}
\int_{\partial \Omega}\left(u n_{a} \nabla^{a} \bar{u}\right) \mathrm{dS} & =\int_{\partial \Omega}\left(u n_{a} \nabla^{a} \bar{u}\right) \mathrm{dS}=\int_{\Omega} \nabla_{a}\left(u \nabla^{a} \bar{u}\right) \mathrm{dV} \\
& =\int_{\Omega}\left(\nabla_{a} u \nabla^{a} \bar{u}+u \Delta \bar{u}\right) \mathrm{dV} \\
& =\int_{\Omega}\left(|\nabla u|^{2}+m^{2}|u|^{2}\right) \mathrm{dV} \geq 0 .
\end{aligned}
$$

To obtain (6) we used that $\Delta u=m^{2} u$ a.e. on $\Omega$, which holds since $\Omega \cap \mathscr{S}_{f}$ has measure zero.

Theorem 1 has applications to quantum field theory. For curved spacetimes which possess both a Riemannian and a Lorentzian section (such as the Schwarzschild black hole), eq. (3) is the inner product in the one-particle space for scalar fields, and the positivity of this inner product is one of the cornerstones of the Euclidean approach. This was discovered by Osterwalder and Schrader [15, 16] for $\mathbb{R}^{d}$, and generalized to curved spacetimes in [9, 10]. From the proof of Theorem 1, we see that $\left\langle f^{\theta}, C f\right\rangle$ is twice the Euclidean action applied to the potential $u=C f$, in the region $\Omega$.

The action functional for a general scalar quantum field theory on a curved background may include a term of the form $\xi R$, where $R$ is the Ricci scalar, and $\xi$ is a real coupling constant. The special case $\xi=0$ is called minimal coupling; we now discuss the general case.

Let $M$ be a complete connected Riemannian manifold with a reflection $\theta$. Let $R$ be the Ricci scalar and $\xi \in \mathbb{R}$ be such that

$$
0<m^{2}+\xi R,
$$

everywhere on $M$. Then $-\Delta+m^{2}+\xi R$ has a self-adjoint extension which is invertible, and thus we define

$$
C_{\xi}=\left(-\Delta+m^{2}+\xi R\right)^{-1} .
$$

Theorem 2. Let $M$ be a complete connected Riemannian manifold with a reflection $\theta$, and assume the curvature bound (7). Let $f$ be as above. Then

$$
0 \leq\left\langle f^{\theta}, C_{\xi} f\right\rangle .
$$

Proof. Following the same steps as leading to (6), we have

$$
\left\langle f^{\theta}, C_{\xi} f\right\rangle=2 \int_{\Omega}\left[|\nabla u|^{2}+\left(m^{2}+\xi R\right)|u|^{2}\right] \mathrm{dV} .
$$

The conclusion follows. 


\section{Comparison of Dirichlet and Neumann Covariance}

Glimm and Jaffe [8, 7] discovered that reflection positivity for free Euclidean fields is equivalent to the operator-monotonicity of the Green's operator $C$, as one varies boundary conditions on the $t=0$ plane. More precisely $C_{D} \leq C_{N}$, where $D, N$ denote respectively the classical Dirichlet or Neumann boundary conditions at $t=0$. The proof remarks that Green's functions satisfying Dirichlet and Neumann boundary conditions can be obtained using mirror charges, and these representations lead to reflection positivity. De Angelis, de Falco, and Di Genova [3] used this property to give a simple proof of reflection positivity for manifolds with an isometric involution $\theta$; we also use this method here.

We first discuss Dirichlet and Neumann conditions on manifolds in general, and then in the special case of a reflection, give a simple proof of the fundamental inequality $C_{D} \leq C_{N}$.

Lemma 3. Let $M$ be complete and connected with a reflection $\theta$. Let $\mathscr{O} \subset M$ be a submanifold with boundary $\partial \mathscr{O} \subseteq M^{\theta}$. Let $C_{D, N}$ denote the resolvent of the Laplace operator on $L^{2}(\mathscr{O})$ with either Dirichlet $(D)$ or Neumann $(N)$ boundary conditions on $\partial \mathscr{O}$. Then

$$
C_{D}=\left(I-U_{\theta}\right) C, \quad C_{N}=\left(I+U_{\theta}\right) C, \quad \text { and } \quad U_{\theta} C=\frac{1}{2}\left(C_{D}-C_{N}\right) \quad \text { on } \quad L^{2}(\mathscr{O}) .
$$

Proof. Write $C$ in integral form with kernel $\mathscr{C}$, so that

$$
(C f)(x)=\int_{M} \mathscr{C}(x, y) f(y) d \operatorname{vol}_{y}
$$

where vol is the Riemannian volume measure; in coordinates $d \operatorname{vol}_{x}=\left(\operatorname{det} g_{a b}\right)^{1 / 2} d x$. Note that $\mathscr{C}: M \times M \rightarrow \mathbb{R}$ is not defined on the diagonal $x=y$. The two fundamental properties of the kernel $\mathscr{C}$ are invariance under the diagonal action of $G \subset G \times G$, and that it is a Green's function. Thus

$$
\mathscr{C}(g x, g y)=\mathscr{C}(x, y) \quad \forall g \in G, \quad \text { and } \quad\left(-\Delta_{x}+m^{2}\right) \mathscr{C}(x, y)=\delta_{x}(y) .
$$

To prove the second property, write

$$
f(x)=\left(\left(-\Delta+m^{2}\right) C f\right)(x)=\int\left(-\Delta_{x}+m^{2}\right) \mathscr{C}(x, y) f(y) d \operatorname{vol}_{y} .
$$

Then by definition, $\left(-\Delta_{x}+m^{2}\right) \mathscr{C}(x, y)=\delta_{x}(y)$ as distributions.

Since $\left[C, U_{\theta}\right]=0$, the integral kernel of $U_{\theta} C$ is $\mathscr{C}(\theta x, y)$. Thus the kernel of $\left(I-U_{\theta}\right) C$ is

$$
k_{-}(x, y):=\mathscr{C}(x, y)-\mathscr{C}(\theta x, y) .
$$

Clearly for $x \in \partial \mathscr{O}$ or $y \in \partial \mathscr{O}$, we have $k_{-}(x, y)=0$, so $k_{-}$satisfies Dirichlet boundary conditions. Also,

$$
\left(-\Delta_{x}+m^{2}\right) k_{-}(x, y)=\delta_{x}(y)-\delta_{x}(\theta y) .
$$

For $x \in \mathscr{O}$, it follows that $\delta_{x} \circ \theta$ vanishes for test functions supported in $\mathscr{O}$, and hence $k_{-}$is the Dirichlet Green's function in $\mathscr{O}$. Now

$$
k_{+}(x, y):=\mathscr{C}(x, y)+\mathscr{C}(\theta x, y)
$$

is also a Green's function in $\mathscr{O}$ for the same reason. Let $\mathscr{C}_{y}(x)=\mathscr{C}(x, y)$ and let $\partial_{n}$ denote the normal derivative in the variable $x$ on the boundary $\partial \mathscr{O}$. Then

$$
\left.\partial_{n}\left(\mathscr{C}_{y}^{\theta}\right)\right|_{p}=-\left.\partial_{n} \mathscr{C}_{y}\right|_{p} \text { for } p \in \partial \mathscr{O}
$$


It follows that $\partial_{n} k_{+}=0$ on $\partial \mathscr{O}$, so $k_{+}$is the Neumann Green's function on $\mathscr{O}$, completing the proof.

We now prove the operator inequality stated previously. The result is known for $M=\mathbb{R}^{d}$, though the proof which has appeared in the literature is complicated due to delicate issues concerning the domains of self-adjoint operators and associated quadratic forms. We present a simpler proof that also generalizes to manifolds.

Theorem 4. Let $M$ be complete and connected with a reflection $\theta$. Let $\mathscr{O}$ be a submanifold with boundary $\partial \mathscr{O} \subseteq M^{\theta}$. Then

$$
C_{D} \leq C_{N} \quad \text { on } \quad L^{2}(\mathscr{O}) .
$$

Proof. By Lemma 3, for $f \in C_{c}^{\infty}(\mathscr{O})$, we have

$$
\left\langle f,\left(C_{N}-C_{D}\right) f\right\rangle=2\left\langle f, U_{\theta} C f\right\rangle .
$$

Now apply Theorem 1 with $\Omega=\mathscr{O}^{c} \cup \partial \mathscr{O}$. The boundary of $\Omega$ is the same as the boundary of $\mathscr{O}$, and the common boundary is contained in $M^{\theta}$. The support $\mathscr{S}_{f}$ of $f$ is disjoint from $\Omega$ (up to sets of measure zero), so Theorem 1 can be applied. Thus (9) is positive and $C_{D} \leq C_{N}$ as desired.

\section{The Dirac Operator}

A certain sense of mystery surrounds Euclidean fermions. It revolves about two issues, the more elementary of which is whether the Euclidean Green's functions are reflection positive. In the case of Pfaffian or determinantal imaginary-time Green's functions, this reduces to the question of reflection positivity for the pair correlation function. In the following, we resolve this question in the affirmative, giving a proof that is at once very simple, and very general; our proof applies to any bundle of Clifford modules over a static manifold.

4.1. Clifford Bundles. Let $M$ be a Riemannian manifold. The Clifford algebra of the cotangent space $T_{x}^{*} M$ (with its natural inner product) will be denoted $\mathrm{Cl}_{x}$, and the association of the vector space $\mathrm{Cl}_{x}$ to the point $x$ defines the Clifford bundle $\mathrm{Cl}(M) \rightarrow M$.

Now suppose $E \rightarrow M$ is a Hermitian vector bundle such that each fiber $E_{x}$ is a Hermitian $\mathrm{Cl}_{x}$-module in a smooth fashion. Let $\Gamma(E)$ denote the space of smooth sections, and let $\Gamma(E ; \mathscr{O})$ denote the space of local sections over an open set $\mathscr{O} \subset M$. Extend the notation to allow $\mathscr{O}$ to be a submanifold with boundary.

Let $E \rightarrow M$ be endowed with a connection $\nabla$. Since $E_{x}$ is a $\mathrm{Cl}_{x}$-module, the inclusion $T_{x}^{*}(M) \subset \mathrm{Cl}_{x}$ gives rise to a natural bundle map $\mathfrak{m}: T^{*} \otimes E \rightarrow E$ called Clifford multiplication. Explicitly, we have a sequence

$$
\Gamma(E) \stackrel{\nabla}{\longrightarrow} \Gamma\left(T^{*} \otimes E\right) \stackrel{\mathfrak{m}}{\longrightarrow} \Gamma(E) .
$$

Denote Clifford multiplication simply by $\xi \cdot v:=\mathfrak{m}(\xi \otimes v)$. Composing the maps (10) gives the Dirac operator

$$
\not \partial=\mathfrak{m} \nabla: \Gamma(E) \longrightarrow \Gamma(E) .
$$

Many computations are facilitated by the use of local coordinates. Let $\mathscr{O}$ be an open subset of $M$ on which we have defined an orthonormal frame $\left\{e_{j}\right\}$ of tangent 
vector fields, and let $\left\{v_{j}\right\}$ denote a dual coframe of 1 -forms. For $\phi \in \Gamma(E ; \mathscr{O})$, the above definitions imply that

$$
\not \partial \phi=\sum_{j} v_{j} \cdot \nabla_{e_{j}} \phi
$$

A Clifford connection on $E$ is a metric connection $\nabla$ that is a derivation with respect to Clifford multiplication, i.e.

$$
\nabla_{X}(v \cdot s)=\left(\nabla_{X} v\right) \cdot s+v \cdot \nabla_{X} s
$$

for a vector field $X$, a 1-form $v$, and a section $s$. In the first term, $\nabla_{X} v$ denotes the Levi-civita connection on $M$, while in the second term $\nabla_{X}$ denotes the connection on $E$.

If $\nabla$ is a Clifford connection on a boundaryless manifold, then $\not \partial$ is a skewsymmetric operator on the domain of smooth, compactly supported sections 18 , Prop. 1.1, p. 246].

4.2. Reflection Positivity. Let $M$ be a complete Riemannian manifold. Further assume $M$ is static; then there are coordinates $\left(x^{i}: 0 \leq i \leq d-1\right)$ such that $\partial / \partial x^{0}$ is a hypersurface-orthogonal Killing field. In many examples from physics, $x^{0}$ plays the role of (Euclidean) time, so we also write $t=x^{0}$.

Corresponding to the local frame $\partial / \partial x^{i}$ is a dual frame of one-form fields, $d x^{i}$. Let $\gamma^{i}$ denote the operator of Clifford multiplication by $d x^{i}$, so that $\gamma^{i}(v)=d x_{i} \cdot v$. Then

$$
\left\{\gamma^{i}, \gamma^{j}\right\}=2 g^{i j} I
$$

where $g^{i j}$ is the inverse metric, and $I$ is the identity on fibers of $E$. Since the coordinate $t=x^{0}$ is determined (up to a constant) by the geometry, the operator $\gamma^{0}$ does have a coordinate-free meaning, whereas in general, $\gamma^{i}$ for $i \neq 0$ are coordinatedependent.

Locally, a static metric takes the form

$$
d s^{2}=F(x) d t^{2}+G_{a b}(x) d x^{a} d x^{b} .
$$

where $F$ and $G_{a b}$ are $t$-independent functions. After an arbitrary choice of a timezero slice $\Sigma=\{t=0\}, M$ has the structure

$$
M=\Omega_{-} \cup \Sigma \cup \Omega_{+}, \quad \partial \Omega_{ \pm}=\Sigma .
$$

Let $\epsilon: \Omega_{+} \rightarrow \Omega_{-}$be the natural reflection map, which in coordinates is given by

$$
\epsilon(t, y)=(-t, y)
$$

where $y$ is a coordinate on $\Sigma$. This induces a pullback map $\epsilon^{*}$ acting on sections of $E$. Let $\vartheta=\gamma^{0} \epsilon^{*}$. Note that

$$
\{\vartheta, \not \partial\}=\left\{\gamma^{0} \epsilon^{*}, \sum_{j} \gamma^{j} \nabla_{e_{j}}\right\}=0 .
$$

To prove (13), note that the $j=0$ term vanishes since $\left\{\epsilon^{*}, \nabla_{e_{0}}\right\}=0$, while the other terms vanish because $\left\{\gamma^{0}, \gamma^{j}\right\}=g^{0 j}=0$.

Theorem 5. Let $E \rightarrow M$ be a holomorphic Clifford bundle with Clifford connection $\nabla$. For a smooth section $\phi \in \Gamma\left(E ; \Omega_{+}\right)$supported in $\Omega_{+}$, we have

$$
0 \leq\left\langle\vartheta \phi,(\not \partial-m)^{-1} \phi\right\rangle \text {. }
$$


Proof. Let $u=(\not \partial-m)^{-1} \phi$ and $u^{\vartheta}=\vartheta u$, so we have

$$
\begin{aligned}
\left\langle\vartheta \phi,(\not \partial-m)^{-1} \phi\right\rangle & =\langle\vartheta(\not \partial-m) u, u\rangle=\langle\vartheta \not \partial u, u\rangle-m\left\langle u^{\vartheta}, u\right\rangle \\
& =-\int_{\Omega_{-}}\left[\left\langle\not \partial u^{\vartheta}, u\right\rangle+m\left\langle u^{\vartheta}, u\right\rangle\right] \\
& =-\int_{\Omega_{-}}\left[\left\langle\not \partial u^{\vartheta}, u\right\rangle+m\left\langle u^{\vartheta}, u\right\rangle+\left\langle u^{\vartheta},(\not \partial-m) u\right\rangle\right] \\
& =-i \int_{\Omega_{-}}\left[\left\langle D u^{\vartheta}, u\right\rangle-\left\langle u^{\vartheta}, D u\right\rangle\right]
\end{aligned}
$$

where we used $\{\vartheta, \not \partial\}=0$, and to obtain (14) from the previous line, we used that $(\not \partial-m) u=0$ on $\Omega_{-}$.

By [18, p. 247], for any sections $\alpha, \beta$ of $E$ we have

$$
\operatorname{div} X=-i[\langle D \alpha, \beta\rangle-\langle\alpha, D \beta\rangle]
$$

where $X$ is the vector field defined by $\langle X, v\rangle=\langle\alpha, v \cdot \beta\rangle_{E}$ for $v \in \Omega^{1}(M)$. Apply this with $\alpha=u^{\vartheta}$ and $\beta=u$ so, using (15), we have

$$
\left\langle\vartheta \phi,(\not \partial-m)^{-1} \phi\right\rangle=\int_{\Omega_{-}} \operatorname{div} X \mathrm{dV},
$$

where $\langle X, v\rangle=\langle\vartheta u, v \cdot u\rangle_{E}$ and $\mathrm{dV}$ is the volume element on $M$.

On $\Sigma$, the outward-pointing unit normal to $\Omega_{-}$is the Killing vector $\partial_{t}$ divided by its norm, i.e. $\hat{n}=F^{-1 / 2} \partial_{t}$, where $F$ is defined in eq. (12). Let $\nu$ denote the 1-form dual to $\hat{n}$, so $\nu=\sqrt{F} d x^{0}$. Then by the divergence theorem,

$$
\int_{\Omega_{-}} \operatorname{div} X \mathrm{dV}=\int_{\partial \Omega_{-}}\langle X, \nu\rangle \mathrm{dS} .
$$

On $\Sigma=\partial \Omega_{-}, \epsilon$ is the identity map and so $u^{\vartheta}=\gamma^{0}(u)$. Also, $\nu \cdot s=\sqrt{F} \gamma^{0}(s)$, so we have

$$
\langle X, \nu\rangle=\langle\vartheta u, \nu \cdot u\rangle_{E}=\left\langle\gamma^{0}(u), \sqrt{F} \gamma^{0}(u)\right\rangle, \quad(\text { on } \Sigma) .
$$

Hence

$$
\left\langle\vartheta \phi,(\not \partial-m)^{-1} \phi\right\rangle=\int_{\Omega_{-}} \operatorname{div} X \mathrm{dV}=\int_{\partial \Omega_{-}}\left\langle\gamma^{0}(u), \sqrt{F} \gamma^{0}(u)\right\rangle \mathrm{dS} \geq 0 .
$$

The power of Theorem 5 lies in its generality; the result is valid for any Clifford connection on any vector bundle over a static manifold. This includes as particular examples the Dirac operator on the spinor bundle $S(\tilde{P})$ over a manifold with a spin structure $\tilde{P} \rightarrow M$, as well as the "twisted Dirac operator" $D_{F}$ on the tensor product $E=S(\tilde{P}) \otimes F$, where $F$ is a bundle with metric connection.

As a corollary to Theorem 5, we infer the existence of a Hilbert space $\mathscr{H}_{D}$ whose inner product is given by

$$
\left(s, s^{\prime}\right)_{D}=\left\langle\vartheta s,(\not \partial-m)^{-1} s^{\prime}\right\rangle .
$$

Precisely, $\mathscr{H}_{D}$ is the completion of the coset space $\Gamma\left(E ; \Omega_{+}\right) / \mathscr{N}_{D}$, where $\mathscr{N}_{D}$ is the kernel of the form $(\cdot, \cdot)_{D}$. The space $\mathscr{H}_{D}$ can be interpreted as the one-particle space for a theory of fermions on the spacetime $M$. 
4.3. Flat Spacetimes. It is very useful to see the abstract framework of the last two sections worked out in the explicit example of $M=\mathbb{R}^{d}$. In this case, we also prove reflection positivity by Fourier analysis.

For an integral operator $C$ on $L^{2}\left(\mathbb{R}^{d}\right)$, we use the convention

$$
C f(x)=\int C(x, y) f(y) d y \text {. }
$$

For $C=\left(-\Delta+m^{2}\right)^{-1}$, the kernel is translation-invariant, so we write $C(x, y)=$ $C(x-y)$, and we may obtain the latter explicitly via the Fourier transform

$$
C(x, y)=C(x-y)=(2 \pi)^{-d} \int \frac{e^{-i p(x-y)}}{p^{2}+m^{2}} d p .
$$

Note that $C(x-y)=\overline{C(y-x)}=C(y-x)$. It follows that the integral kernel of $\partial_{x} C$ is equal to $\partial_{x} C(x-y)$.

Let $\gamma_{j}$, for $j=0, \ldots, d-1$, be a set of Hermitian operator $Z^{2}$ on a complex Hilbert space $V$ satisfying:

$$
\left\{\gamma_{i}, \gamma_{j}\right\}=2 \delta_{i j} I
$$

Denote $\not p=\sum_{i=0}^{d-1} \gamma_{i} p_{i}$, with $\not \partial$ defined similarly. This arises from the general theory of Section 4.1, by setting $E=\mathbb{R}^{d} \times V$, a trivial Hermitian vector bundle over $\mathbb{R}^{d}$ with standard Riemannian and Clifford structures. Then

$$
\not p^{2}=\frac{1}{2}\{\not p, \not p\}=\frac{1}{2} \sum_{a, b} p_{a} p_{b}\left\{\gamma_{a}, \gamma_{b}\right\}=p^{2} I .
$$

Similarly, $\not{ }^{2}=\Delta$, so $-(\not \partial+m)(\not \partial-m)=-\Delta+m^{2}=C^{-1}$ and hence

$$
(\not \partial-m)^{-1}=-(\not \partial+m) C .
$$

Let $\epsilon\left(x_{0}, \vec{x}\right)=\left(-x_{0}, \vec{x}\right)$ be a coordinate reflection. For $f: \mathbb{R}^{d} \rightarrow V$, define

$$
(\vartheta f)(x):=\gamma^{0} f(\epsilon x) \text {. }
$$

It follows that $\vartheta$ is a self-adjoint operator on $L^{2}\left(\mathbb{R}^{d}, V\right)$ with $\vartheta^{2}=I$.

Theorem 6. The operator $(\not \partial-m)^{-1}$ is reflection-positive in the sense that

$$
\left\langle\vartheta f,(\not \partial-m)^{-1} f\right\rangle \geq 0 \quad \text { for } \quad \operatorname{supp} f \subseteq\left\{x_{0}>0\right\} .
$$

We give two proofs of Theorem 6, one by Fourier analysis and one by potential theory.

Proof (Fourier analysis). By direct calculation,

$$
-\gamma^{0}(\not \partial+m) C(x)=-\gamma^{0} \int d p_{0} d \vec{p}\left(\gamma^{0}\left(-i p_{0}\right)+\sum_{j>0} \gamma^{j}\left(-i p_{j}\right)+m\right) \frac{e^{-i p x}}{p^{2}+m^{2}},
$$

By contour integration, for any $t \in \mathbb{R}$ we have:

$$
\int \frac{e^{-i p_{0} t}}{p_{0}^{2}+\omega^{2}} d p_{0}=\frac{\pi e^{-|t| \omega}}{\omega}, \quad \int p_{0} \frac{e^{-i p_{0} t}}{p_{0}^{2}+\omega^{2}} d p_{0}=-i \pi e^{-|t| \omega}
$$

\footnotetext{
${ }^{2}$ An example in $d=4$ is $\gamma_{0}=\left(\begin{array}{cc}0 & I \\ I & 0\end{array}\right)$, and $\gamma_{j}=i\left(\begin{array}{cc}0 & \sigma_{j} \\ -\sigma_{j} & 0\end{array}\right)$ for $j=1,2,3$.
} 
We use these formulas with $\omega=\left(\vec{p}^{2}+m^{2}\right)^{1 / 2}$, and $t=x_{0}$. So,

$$
\begin{aligned}
-\gamma^{0}(\not \partial+m) C(x) & =\pi \int\left(e^{-|t| \omega}+\frac{e^{-|t| \omega}}{\omega}\left[\sum_{j=1}^{d-1} i \gamma_{0} \gamma^{j} p_{j}-m \gamma_{0}\right]\right) e^{-i \vec{p} \cdot \vec{x}} d \vec{p} \\
& =\pi \int \frac{e^{-|t| \omega}}{\omega} A e^{-i \vec{p} \cdot \vec{x}} d \vec{p},
\end{aligned}
$$

where we define

$$
\vec{\eta}:=i \gamma^{0} \vec{\gamma}, \quad \omega=\left(\vec{p}^{2}+m^{2}\right)^{1 / 2}, \quad \text { and } \quad A=\omega I+\vec{\eta} \cdot \vec{p}-m \gamma_{0} .
$$

Here, each component $\eta_{j}$ and $A$ are $d \times d$ matrices, and $A$ has $\vec{p}$-dependent matrix elements. The matrix $\Omega=\vec{\eta} \cdot \vec{p}-m \gamma_{0}$ is Hermitian with $\Omega^{2}=\omega^{2} I$, hence $A=\omega I+\Omega$ has eigenvalues $0,2 \omega$. In particular, $A$ is a positive matrix. The rest of the proof depends only on the property $A \geq 0$ and not on the details of $A$. To complete the argument, we now have

$$
\begin{aligned}
\left\langle\vartheta f,(\not \partial-m)^{-1} f\right\rangle & =\left\langle\epsilon^{*} f, \gamma_{0}(\not \partial-m)^{-1} f\right\rangle \\
& =-\int_{x_{0}<0} d x \int_{y_{0}>0} d y\left\langle f\left(-x_{0}, \vec{x}\right), \quad\left[\gamma_{0}\left(\not \partial_{x}+m\right) C\right](x-y) f(y)\right\rangle \\
& =-\int_{x_{0}<0} d x \int_{y_{0}>0} d y \int d \vec{p}\left\langle f\left(-x_{0}, \vec{x}\right), A \frac{e^{-\left|x_{0}-y_{0}\right| \omega}}{\omega} e^{-i \vec{p} \cdot(\vec{x}-\vec{y})} f(y)\right\rangle \\
& =\int d \vec{p}\left|\int e^{i \vec{p} \cdot \vec{x}-x_{0} \omega}\left(\frac{A}{\omega}\right)^{1 / 2} f(x) d x\right|^{2} \geq 0 .
\end{aligned}
$$

Proof (potential theory). We will now give a second proof of (18), which follows the proof of Theorem 5. Rather than integrating out $p_{0}$ in the Fourier transform, we will instead use integration by parts to reduce the expression to a boundary term. Note that $\{\vartheta, \not \supset\}=0$, as may be proved directly, or deduced as a special case of (13).

Define $u=(\not \partial-m)^{-1} f$ and let $u^{\vartheta}=\vartheta u=\gamma^{0} \epsilon^{*} u$. Then

$$
\begin{aligned}
\left\langle\vartheta f,(\not \partial-m)^{-1} f\right\rangle & =\int_{\Omega_{-}}\langle\vartheta(\not \partial-m) u, u\rangle d x=-\int_{\Omega_{-}}\left[\left\langle\not \partial u^{\vartheta}, u\right\rangle+m\left\langle u^{\vartheta}, u\right\rangle\right] d x \\
& =-\int_{\Omega_{-}}\left[\left\langle\not \partial u^{\vartheta}, u\right\rangle+\left\langle u^{\vartheta},(\not \partial-m) u\right\rangle+m\left\langle u^{\vartheta}, u\right\rangle\right] d x \\
& =-\int_{\Omega_{-}}\left[\left\langle\not \partial u^{\vartheta}, u\right\rangle+\left\langle u^{\vartheta}, \not \partial u\right\rangle\right] d x
\end{aligned}
$$

To obtain (19), we used that $(\not \partial-m) u=f$ is zero on $\Omega_{-}$. 3

Now perform integration by parts on the first term in (20), moving the $\not \partial$ onto the $u$. All of the non-surface terms cancel. There is no boundary in the spatial directions, so we only need to compute the boundary term which occurs at the $t=0$ plane. To do this, consider

$$
-\int_{0}^{\infty} d x_{0}\left\langle\gamma^{0} \partial_{0}(\vartheta u), u\right\rangle=\int_{x_{0}=0}|u|^{2} d^{d-1} x+\text { (non-surface terms) . }
$$

\footnotetext{
${ }^{3}$ It is interesting that in (19), the explicit mass term cancels out. Thus all of the $m$-dependence is contained in $u$, which depends implicitly on $m$ through the equation $(\not \partial-m) u=f$.
} 
Here we used that $\vartheta u=\gamma^{0} u$ on the boundary, and $\left(\gamma^{0}\right)^{2}=I$. Then

$$
\left\langle\vartheta f,(\not \partial-m)^{-1} f\right\rangle=\int_{x_{0}=0}|u|^{2} d^{d-1} x \geq 0 .
$$

The resulting formula for the fermionic inner product is the special case of (16) with $F=1$.

\section{Further Directions}

A more subtle question arises when one asks whether one can obtain a representation of these Euclidean Green's functions as expectations of "classical" Euclidean fields. Berezin proposed some time ago that classical fermion fields take values in a Grassmann algebra.

Osterwalder and Schrader demonstrated that one can have Euclidean Dirac fields. But they showed that one must double the number of degrees of freedom; in this way they introduced a Euclidean Dirac field $\Psi$ that is independent from (anti-commutes with) its Dirac adjoint field $\bar{\Psi}$. The existence of a representation of the Dirac propagator as an expectation of products of Euclidean fields on curved spacetimes is, at present, an open question. The results in this paper show that a representation in terms of Euclidean Fermi fields is a reasonable thing to expect.

\section{REFERENCES}

[1] Dmitri V. Alekseevsky, Andreas Kriegl, Mark Losik, and Peter W. Michor. Reflection groups on Riemannian manifolds. Ann. Mat. Pura Appl. (4), 186(1):25-58, 2007.

[2] Isaac Chavel. Riemannian geometry, volume 98 of Cambridge Studies in Advanced Mathematics. Cambridge University Press, Cambridge, second edition, 2006. A modern introduction.

[3] Gian Fabrizio De Angelis, Diego de Falco, and Glauco Di Genova. Random fields on Riemannian manifolds: a constructive approach. Comm. Math. Phys., 103(2):297-303, 1986.

[4] J. Dimock. Markov quantum fields on a manifold. Rev. Math. Phys., 16(2):243$255,2004$.

[5] J. Fröhlich. Unbounded, symmetric semigroups on a separable Hilbert space are essentially selfadjoint. Adv. in Appl. Math., 1(3):237-256, 1980.

[6] Matthew P. Gaffney. The harmonic operator for exterior differential forms. Proc. Nat. Acad. Sci. U. S. A., 37:48-50, 1951.

[7] James Glimm and Arthur Jaffe. A note on reflection positivity. Lett. Math. Phys., 3(5):377-378, 1979.

[8] James Glimm and Arthur Jaffe. Quantum physics. Springer-Verlag, New York, second edition, 1987. A functional integral point of view.

[9] Arthur Jaffe and Gordon Ritter. Quantum field fheory on curved backgrounds. II. the Euclidean functional integral. Comm. Math. Phys., 270(2):545-572, 2007.

[10] Arthur Jaffe and Gordon Ritter. Quantum field theory on curved backgrounds. II. spacetime symmetries. submitted, 2007. [arXiv:0704.0052v1].

[11] Tosio Kato. Perturbation theory for linear operators. Classics in Mathematics. Springer-Verlag, Berlin, 1995. Reprint of the 1980 edition. 
[12] Abel Klein and Lawrence J. Landau. Construction of a unique selfadjoint generator for a symmetric local semigroup. J. Funct. Anal., 44(2):121-137, 1981.

[13] Edward Nelson. Construction of quantum fields from Markoff fields. J. Functional Analysis, 12:97-112, 1973.

[14] Edward Nelson. The free Markoff field. J. Functional Analysis, 12:211-227, 1973.

[15] Konrad Osterwalder and Robert Schrader. Axioms for Euclidean Green's functions. Comm. Math. Phys., 31:83-112, 1973.

[16] Konrad Osterwalder and Robert Schrader. Axioms for Euclidean Green's functions. II. Comm. Math. Phys., 42:281-305, 1975. With an appendix by Stephen Summers.

[17] K. Symanzik. Euclidean quantum field theory. I. Equations for a scalar model. J. Mathematical Phys., 7:510-525, 1966.

[18] Michael E. Taylor. Partial differential equations. II, volume 116 of Applied Mathematical Sciences. Springer-Verlag, New York, 1996. Qualitative studies of linear equations.

Harvard University, 17 Oxford St., Cambridge, MA 02138

E-mail address: Arthur_Jaffe@harvard.edu, ritter@post.harvard.edu 